AperTO - Archivio Istituzionale Open Access dell'Università di Torino

\title{
Point defects in electron paramagnetic resonance
}

\section{This is a pre print version of the following article:}

Original Citation:

Availability:

This version is available http://hdl.handle.net/2318/1574663

since 2016-06-28T16:36:23Z

Published version:

DOI:10.1007/978-3-319-14367-5_10

Terms of use:

Open Access

Anyone can freely access the full text of works made available as "Open Access". Works made available under a Creative Commons license can be used according to the terms and conditions of said license. Use of all other works requires consent of the right holder (author or publisher) if not exempted from copyright protection by the applicable law. 
This is the author's final version of the contribution published as:

Giamello, Elio; Chiesa, Mario; Paganini, Maria Cristina. Point defects in electron paramagnetic resonance. SPRINGER SERIES IN SURFACE SCIENCES. 58 pp: 303-326.

DOI: $10.1007 / 978-3-319-14367-5 \_10$

The publisher's version is available at:

http://link.springer.com/content/pdf/10.1007/978-3-319-14367-5_10

When citing, please refer to the published version.

Link to this full text:

http://hdl.handle.net/None 


\title{
Point Defects in Electron Paramagnetic Resonance
}

\author{
Elio Giamello, Mario Chiesa, Maria Cristina Paganini \\ Università di Torino, Dipartimento di Chimica e NIS, Via P. Giuria 7 \\ 10125 Torino Italy.
}

\begin{abstract}
Electron Paramagnetic Resonance (EPR) has played a paramount role in understanding the nature and the main features of point defects in the bulk of both ionic and covalent solids. In the present chapter, after a brief introduction to the technique, the employ of EPR to unravel the surface counterparts of bulk point defects in the case of metal oxides will be illustrated with particular attention to the defects capable of chargecarriers trapping.
\end{abstract}

\section{Introduction}

Solid State Physics, Solid State Chemistry and Material Science have a strategic interest in methods allowing to understand the nature, location and electronic structure of point defects. More than fifty years of research in these fields have demonstrated that, among all physical methods, Electron Paramagnetic Resonance (EPR) techniques are extremely powerful tools for describing, in particular, point defects in the bulk of solids and play an important role also in picturing surface localized defects. EPR is a magnetic resonance technique capable of monitoring paramagnetic centres (i.e. centres bearing one or more unpaired electrons) in a given system. The important role of EPR is due to two basic factors, namely the intrinsic accuracy of EPR techniques in describing structure and electron spin density distribution of a (paramagnetic) species and the high sensitivity of the techniques which allows detection of tiny concentrations of paramagnetic centres. Additionally EPR is, nowadays, easily matched with advanced, DFT-based theoretical techniques capable of accurate computations of the spectral parameters. The intrinsic limitation of EPR which cannot be applied to diamagnetic systems is, in this case, attenuated by the fact that many and highly important point defects in solid materials are paramagnetic. In the present chapter, after a short introduction to the EPR techniques, the attention will be preliminarily paid to the paramagnetic point defects present in the bulk of ionic solids in order to examine in more detail their counterparts present at the surface of metal oxides. 


\section{The EPR techniques: a summary.}

The term Electron Paramagnetic Resonance (EPR) encompasses a family of techniques based on the absorption of electromagnetic radiation in the microwave frequency region by a paramagnetic sample placed in a magnetic field. Since the first experiment in 1945, the conventional Continuous Wave-EPR (CW-EPR) has been dominant in the first fifty years of applications to Chemistry, Physics and Material Science studies. In a CW-EPR experiment the sample under investigation interacts with a homogeneous magnetic field varying in a selected range and is irradiated by a continuous flow of microwaves at fixed frequency which, at a particular value of magnetic field when resonance conditions are fulfilled entails a transition between two spin states. Following what previously done in Nuclear Magnetic Resonance, pulsed methods were also, more recently, introduced in EPR. These techniques, based on irradiation by controlled pulses of microwaves, are finding wider applications in many fields. Continuous wave techniques include CW-EPR, ENDOR (Electron Nuclear Double Resonance) and HF-EPR (High Field Electron Paramagnetic Resonance) while, among the many pulsed methods, we can find FS-ESE (Field Swept Electron Spin Echo), ESEEM (Electron Spin Echo Envelope Modulation) or FT-ESR (Fourier Transform Electron Spin Resonance) and pulsed ENDOR [1]. In addition to the above mentioned techniques the methods of optically and electrically detected EPR (ODEPR and EDEPR) have proven to be particularly important for the structural analysis of point defects in solids thank in particular to the much increased sensitivity (several order of magnitudes) and selectivity with respect to conventional EPR. For an excellent review on the applications of these methods to the determination of microscopic defect structures, the reader is referred to the book by Spaeth and Overhof. [2]

\subsection{The spin-Hamiltonian formalism.}

The electron spin resonance experiment is the observation of the energy required to reverse the direction of an electron spin in the presence of an external magnetic field. When immersed in the lines of force of a static magnetic field, in fact, the two spin states lose their degeneracy (Zeeman effect) and become separated by an energy depending on the static magnetic field $\mathbf{B}$ whose induction is measured in Tesla $(T)$ or Gauss $(G)(1 \mathrm{~T}=$ $\left.10^{4} \mathrm{G}\right)$. Electrons in the lower Zeeman state $(\beta)$ can be promoted in the upper state $(\alpha)$ by absorption of a quantum of electromagnetic radiation energy usually in the region of microwaves, provided that the quantum energy $h v$ coincides with the energy difference between the $\alpha$ and $\beta$ states. For a free electron one has 


$$
h v=g \mu_{B} B
$$

where $v$ is the radiation frequency, $g_{\mathrm{e}}$ is the free electron $g$ value $\left(g_{\mathrm{e}}=2.002319\right), \mu_{\mathrm{B}}$ is the atomic unit of magnetic moment called Bohr magneton $\left(\mu_{\mathrm{B}}=9.274 \cdot 10^{-24} \mathrm{~J} \cdot \mathrm{T}^{-1}\right)$. Equation (1) is the fundamental equation of EPR spectroscopy.

A typical Continuous Wave (CW) EPR spectrum reports the first derivative (vide infra) of the microwave absorption as a function of the magnetic field in a selected range of the latter. The EPR spectrum of a paramagnetic species can be described by the spin Hamiltonian (2) which defines the main energy terms as follows:

$$
\mathrm{H}=\mathrm{H}_{E Z}+\mathrm{H}_{F}+\mathrm{H}_{H F S}
$$

The first term is the electronic Zeeman operator $\left(\mathrm{H}_{E Z}=\mu_{\mathrm{B}} \boldsymbol{S} \cdot \boldsymbol{g} \cdot \boldsymbol{B}\right)$ which accounts for the interaction of the electron spin $\boldsymbol{S}$ with the external magnetic field $\boldsymbol{B}$. In a real system, different from a free electron, $g$ shifts from the $g_{\mathrm{e}}$ value and is no more, usually, a scalar. The $\boldsymbol{S}-\boldsymbol{B}$ interaction, in fact, is gauged by the $\boldsymbol{g}$ tensor, a $3 \times 3$ matrix which, in general, can be reduced to its diagonal form with the principal elements $g_{x x}, g_{y y}, g_{z z}$ put into evidence. The values of the g elements depend on the electronic structure (ground and excited states) of the paramagnetic species.

The fine structure term $\left(\mathrm{H}_{\mathrm{F}}=\boldsymbol{S} \cdot \boldsymbol{D} \cdot \boldsymbol{S}\right)$ describes the interaction between two or more unpaired electrons through the $\boldsymbol{D}$ tensor. This term is zero in the case of $S=1 / 2$ i.e. for the centers having a single unpaired electron.

The third term ( $\left.\mathrm{H}_{\mathrm{HFS}}=\boldsymbol{S} \cdot \boldsymbol{A} \cdot \boldsymbol{I}\right)$ represents the hyperfine interaction between the electron spin and nuclear spins. $\boldsymbol{A}$ is the hyperfine tensor and $\boldsymbol{I}$ is the nuclear spin vector. In CW-EPR the hyperfine interactions give rise to line splitting in the spectrum (the hyperfine structure). $2 l+1$ lines are expected for the interaction of the electron spin with a nucleus having nuclear spin quantum number I. $\boldsymbol{A}$ is composed of two main contributions, i.e. the isotropic Fermi contact term (a scalar arising from the finite probability of the electron being located at the position of the nucleus, which has non-zero values in case of s-orbitals only) and the anisotropic electron-nucleus dipolar coupling expressed by a matrix $T$. The hyperfine interaction is extremely important in EPR in that the values of the hyperfine constants (derived analyzing the separation of the spectral lines) provide information on the degree of localization/delocalization of the unpaired electron wave function and on the chemical environment of the unpaired electron center provided that magnetic nuclei ( i.e. nuclei with I $\neq 0$ ) are present in the surroundings of the center. In some particularly favorable cases a complete map of the spin density can be derived from the hyperfine tensors. Few other 
terms of the Spin-Hamiltonian are not discussed here as they are less important than those reported in Equation 1 and affect very weakly the EPR spectra discussed in the present document.

The EPR tensors can be classified on the basis of their symmetry (for instance, an isotropic tensor has three equal principal components, an axial tensor has two equal components differing from the third one, a rhombic tensor has three distinct components) which in turn depends on the point symmetry of the paramagnetic centre. Paramagnetic centers located at solid surfaces are submitted to some symmetry restrictions In the three most symmetric cases mentioned above (isotropic, axial and rhombic) the axes of $\boldsymbol{g}$ and $\boldsymbol{A}$ tensors coincide while in the less symmetric case cases they do not. Due to the phase sensitive detection systems employed in CW-EPR spectroscopy the output is usually the first derivative of the microwave absorption reported as a function of the scanned field.

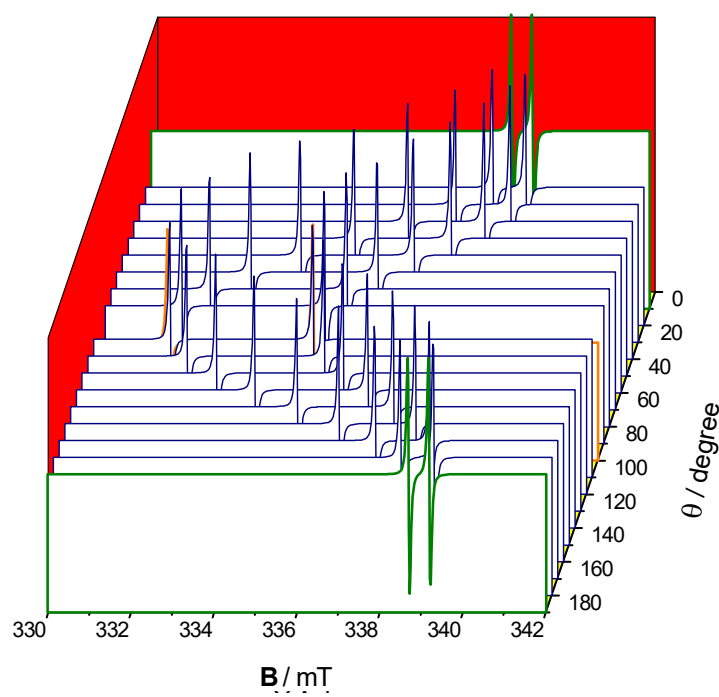

a)

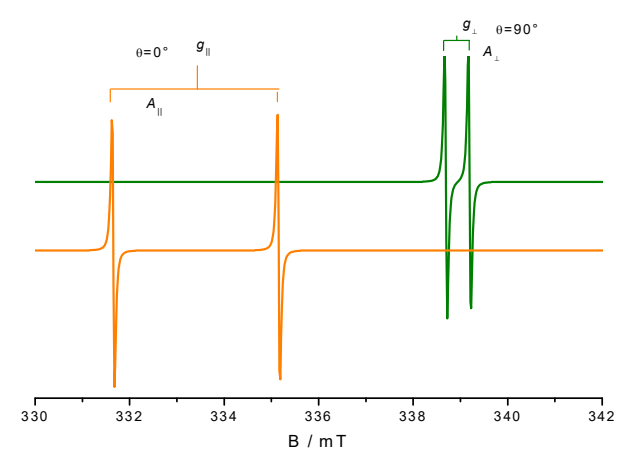

b)

Fig. 1. a) Angular dependence of the EPR signal of an $S=1 / 2-I=1 / 2$ species with uniaxial symmetry in a single crystal. The spectrum consists in a pair of lines. According to the orientation a continuous variation of the centre of the pair ( $\mathbf{g}$ anisotropy) and of the line splitting (A anisotropy) is observed. b) EPR signals of the same species at two particular orientations: $\theta=0$ and $\theta=\pi / 2$.

\subsection{Single crystal systems.}

The presence of tensors in all terms of equation (2) reflects the anisotropy of magnetic interactions. In pragmatic terms, this means that, when the paramagnetic system is located into a single crystal, the EPR signal changes according to the orientation of the crystal in the external magnetic field $\boldsymbol{B}$. In a classic CW-EPR spectrum the values of the $\boldsymbol{g}$ tensor elements determine the position of the resonant field $B_{\text {res }}$ while those of $\boldsymbol{A}$ determine the amplitude $A$ of the hyperfine splitting. Both $B_{\text {res }}$ and $A$ depend on the orientations (Figure 
1). Single crystal EPR spectra are usually recorded with the paramagnetic crystal mounted so that it can be rotated in the resonant cavity about one of the reference axes. After repeating this procedure in each of the coordinate planes, one then seeks to fit the observed curves with the theoretical formulas by adjusting the relevant parameters (i.e.the $\mathbf{g}$ and $\mathbf{A}$ components). EPR measurements of single crystal surfaces or thin films are now available in the literature $[3,4,5]$. The number of EPR experiments on single crystal surfaces is certainly growing, however the large majority of published work refers to polycrystalline systems or powders [6].

\subsection{Polycrystalline systems.}

Polycrystalline powders are disordered systems composed of many small crystallites randomly oriented in space. The resultant EPR spectrum, called a powder spectrum, is the envelope of spectra corresponding to all possible orientations of the paramagnetic species with respect to the applied magnetic field (Figure 2). The simultaneous presence of resonance in the whole range between $B_{\min }$ and $B_{\max }$ does not create a uniform envelope, because the resonant lines are not uniformly distributed over this range. In real cases, CWEPR powder spectra of surface centers often provide challenging problems, such as heterogeneity of surface sites, presence of different paramagnetic species with overlapping signals, line broadening due to interaction between nearby paramagnetic species etc. Some of the reported complications can be overcome using advanced EPR techniques such as ENDOR or pulsed EPR.
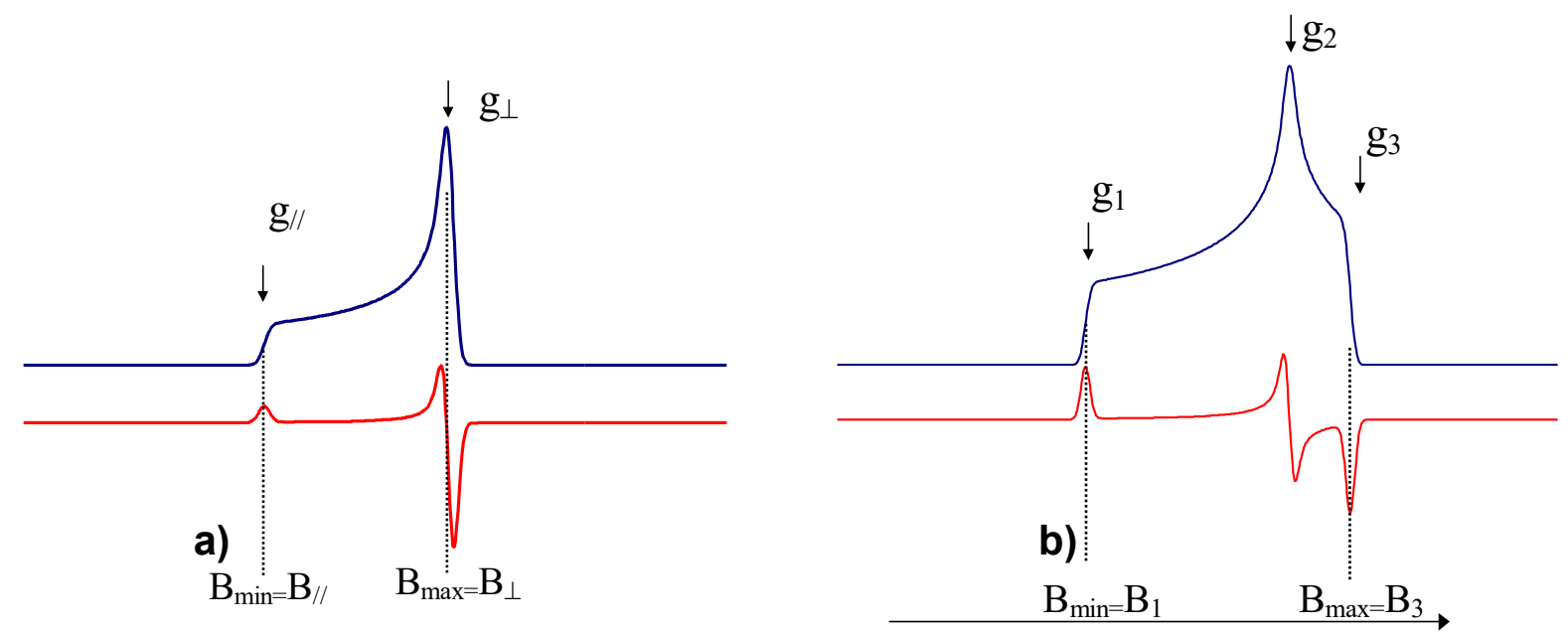

Fig. 2. Simulated EPR powder spectra of two species with $S=1 / 2, l=0$ and having axial (a) and rhombic (b) symmetry (and no hyperfine structure) 
However, even in the case of classic CW-EPR one frequently encounters sufficiently well resolved powder spectra from which, also with the systematic use of tools like multifrequency EPR, isotopic substitution and computer simulation one can achieve valuable information.

\section{Colour centres in the bulk of ionic solids.}

In ionic solids, and in metal oxides in particular, the zero-dimensional defects or point defects are the most important ones. These are essentially based on single or multiple ion vacancies (Schottky defects) and on interstitial ions (Fraenkel defects). The EPR technique is not intrinsically able to monitor ion vacancies, however it has been possible to take advantage of the fact that the vacancies are often populated (or can be artificially populated) by charge carriers to investigate the features of the defects when they achieve, localizing electrons or holes, a paramagnetic state. The study of paramagnetic point defects has been therefore of interest not only for solid state physics but also for other fields such as radiation chemistry, photochemistry and solid state chemistry in that the presence of charge carriers into ion vacancies is commonly related to the interaction of the solid with ionizing radiations or to chemical modifications of the solid itself.

Ionic insulating solids, which are formed by regular arrays of ions having alternating electric charge and generating strong electric potential within the matrix, are suited to host localized charge carriers. The simplest systems containing electrons are indeed the color centers formed in ionic solids. These systems basically consists in one or more electrons trapped in the volume of an anion vacancy (Fig. 3). Color centers can be found in naturally occurring ionic crystals or can be generated in unperturbed crystals by high energy irradiation or chemical treatments (metal addition). The presence of such centres origins energy states in the band gap of the insulating solid which are able to absorb visible light thus colouring the crystals. For this reason they were called colour centres or F-centers. Alkali halides and alkali-earth oxides are the solids in which colour centres have been most frequently found and investigated. The model for the colour centres in a solid having rock-salt structure, consists in an anion vacancy surrounded by six positive ions in octahedral symmetry which can trap one or two electrons according to the nature of the solid. This model sketched in Figure 3 is due to De Boer [7] (1937) and, in spite of the very intense experimental investigation by various physical methods, it was confirmed about 20 years later only after the advent of electron magnetic resonance methods (EPR 
and ENDOR). As the De Boer picture for $F$ centres is very close to the ideal model of a particle in a box, the $\mathrm{F}$ centres became an important reference for solid state physics. Among the various cases of electrons observed by EPR in the solid state, colour centres differ from all other types of localized and mobile electrons. In fact, in spite of the fact that the major fraction of the electron spin density is found within the volume of the vacancy, the ground state wave function of the electron exhibits a tail which causes the electron to interact with a surprisingly high number of ionic shells surrounding the vacancy.

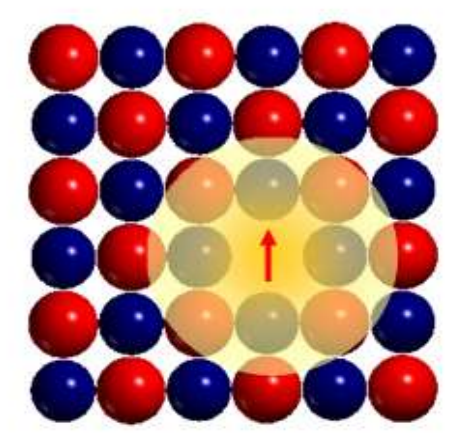

Figure 3. Bidimensional scheme of a F centre in a crystal having rock-salt structure.

Under the point of view of EPR this fact origins the formation, for $F$ centres in alkali halides, of a rich and very complex hyperfine structure due to the fact that all alkali metals and halogens nuclei have non zero nuclear spin. In few cases ( $\mathrm{LiF}, \mathrm{NaF}, \mathrm{RbCl}, \mathrm{CsCl}$ ) the hyperfine structure of the EPR spectrum is partially resolved (Figure 4) in all other cases (eg $\mathrm{LiCl}, \mathrm{NaCl}, \mathrm{NaBr}, \mathrm{KCl}, \mathrm{Kl}$ etc.) the spectrum, due to the huge number of hyperfine lines present in a limited range of magnetic field, consist in a broad unstructured Gaussian line whose width ranges from few tenths to some hundreds Gauss from case to case. In these cases the hyperfine constants become measurable by ENDOR. With this technique a series of lines are observed and can be assigned to the interaction of either $\mathrm{K}^{+}$or $\mathrm{Br}$ ions, belonging to progressively outer coordination spheres, with the trapped electron [8]. Colour centres based on bulk oxygen vacancies have been investigated also in the case of ionic oxides and in particular of alkali-earth oxides. In this case, however, the information is not so rich as for alkaline halides since the presence of magnetic nuclei in these systems is lower (see below). Already in 1968 Henderson and Wertz published the first spectrum of a $\mathrm{F}^{+}$centre in $\mathrm{MgO}$ [9]. 


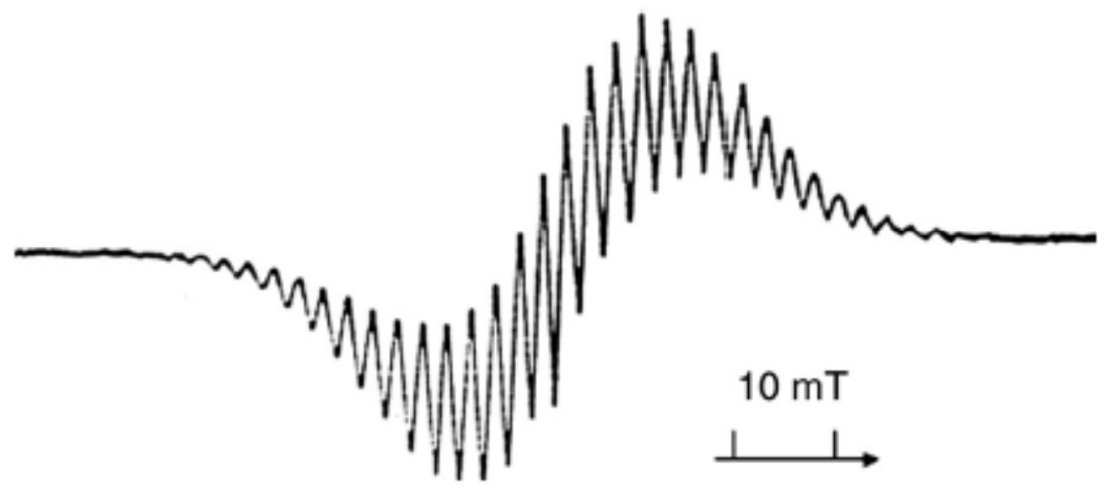

Figure 4. CW-EPR spectrum of $\mathrm{F}$ centres in a single crystal of LiF. The complex hyperfine structure is due to the interaction of the unpaired electron with ${ }^{7} \mathrm{Li}(I=3 / 2)$ and ${ }^{19} \mathrm{~F}(I=1 / 2)$ nuclei. Figure adapted from Kaplan $\mathrm{R}$, Bray PJ (1963) Phys Rev, 129, 1919.

An $\mathrm{F}$ center in the bulk of $\mathrm{MgO}$ or of another alkaline earth oxide consists of one $\left(\mathrm{F}^{+}\right)$or two (F) electrons trapped in an anionic vacancy surrounded by six cationic $\mathrm{Me}^{2+}$ sites in octahedral symmetry. The only $\mathrm{F}^{+}$center is paramagnetic as it contains an odd electron. Both the two-electrons $\mathrm{F}$ center and the simple, unpopulated, $\mathrm{O}^{2-}$ vacancy, which can be indicated as $\mathrm{F}^{2+}$, are not observed by EPR as they are diamagnetic. An electron trapped in an anion vacancy will have at its nearest neighbors mostly ${ }^{24} \mathrm{Mg}$ and ${ }^{26} \mathrm{Mg}$ cations since their combined natural abundance is $89.9 \%$. The ${ }^{25} \mathrm{Mg}$ nuclide constitutes the remaining $10.1 \%$ and has a nuclear spin I of 5/2, while for the other two Mg isotopes the nuclear spin is zero. The EPR spectrum of $\mathrm{F}^{+}$centers in the bulk of a $\mathrm{MgO}$ single crystal is relatively simple. The probability of having magnetic or non magnetic nuclei as nearest neighbors cations can be easily calculated.

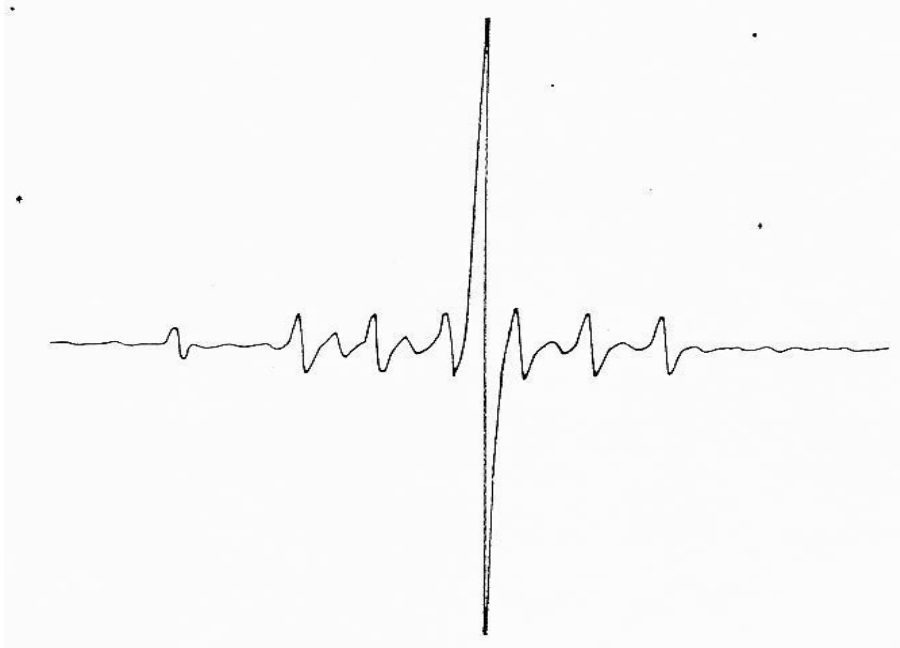

Fig. 5. CW-EPR spectrum of $\mathrm{F}^{+}$centres in a single crystal of $\mathrm{MgO}$. The hyperfine structure (six lines) around the central line is due to the fraction of centres that have one ${ }^{25} \mathrm{Mg}^{2+}$ cation $(I=5 / 2)$ in the six-ion first coordination sphere. Adapted from Henderson B, Wertz J E (1968) Adv Phys 17, 749. 
In particular 0.52 of all trapped electrons will have only ${ }^{24} \mathrm{Mg}$ or ${ }^{26} \mathrm{Mg}$ in the first coordination sphere. These centers give rise to a single symmetric line in a monocrystal whose position (resonant field) varies according to the orientation. For such centers there is no hyperfine structure. Of the remaining $\mathrm{F}^{+}$centers the fraction 0.36 will have one ${ }^{25} \mathrm{Mg}^{2+}$ neighbor (producing a sextet of equally spaced and equally intense lines since the number of expected transitions is $2 \mathrm{I}+1=6$ ) and 0.1 will have two ${ }^{25} \mathrm{Mg}^{2+}$ cations (giving rise to an 11 line hyperfine pattern with well defined intensity distribution)[10]. The EPR spectrum, defined by the authors as "beautifully resolved and unambiguous", is reported in Fig. 5. The 11 line pattern (here not reported) is visible only amplifying the spectrum. The separation of the hyperfine lines monitors the interaction of the trapped electron with the ions of the first coordination sphere.The symmetry and the features of the hyperfine lines fully confirm the de Boer model of colour centres describing an electron trapped in a symmetric box constituted by six magnesium ions.

\section{Localized holes and $\mathbf{V}$ centers in ionic solids.}

Trapped hole centres are electron deficient centres formed by removal of electrons in a band. Removal of an electron leaves behind a net positive charge, i.e. a positive electron hole, or more simply a hole. Electron holes are common defects of the solid state. They can be formed by ionizing radiation or can be induced by chemical modification of a crystal. In chemical terms, and referring to an ionic solid, an electron is ionized from an anion site $\left(\mathrm{Cl}^{-}, \mathrm{O}^{2-}\right)$ leaving an hole on this site. Several ways can be found to stabilize the hole and, if stabilisation occurs, the hole centre is usually observable by EPR. A rich family of such defects, also called $V$ centres has been identified in ionic solids [10]. As opposite to the localized electron, which can be stabilized in the volume of a anion vacancy ( $F$ centre), holes formed in ionic solids cannot stabilize in a corresponding manner (that is in the volume of a cation vacancy) but they tend, instead, to localize in an atomic (or molecular) orbital of the system. In other words, the antimorph of an F centre does not exist. In the case of ionic oxides, which contains divalent $\mathrm{O}^{2-}$ anions (alkaline earth oxides are by far the most investigated materials of this class) an hole, after its formation, will migrate freely until it becomes trapped at a suitable defective site. It is quite common the trapping at a site of net negative charge such as a cation vacancy (Figure 6 ). In this case the defect is known as a $\mathrm{V}^{-}$centre. The negative charge in this notation does not indicate the charge of the carrier but, rather, the whole charge of the defect since a cation vacancy 
locally generates a double negative charge (only partially compensated by the incoming positive hole). In the more exhaustive Kröger and Vink notation the $\mathrm{V}^{-}$center is indicated (considering for example the case of $\mathrm{MgO}$ ) as $\mathrm{V}_{\mathrm{Mg}}$. The positive hole in ionic oxides localizes in the orbitals of one of the oxygen anions surrounding the defect. In chemical terms a diamagnetic $\mathrm{O}^{2-}$ ion transforms into a paramagnetic $\mathrm{O}^{-}$one. This description is fully supported by EPR data.

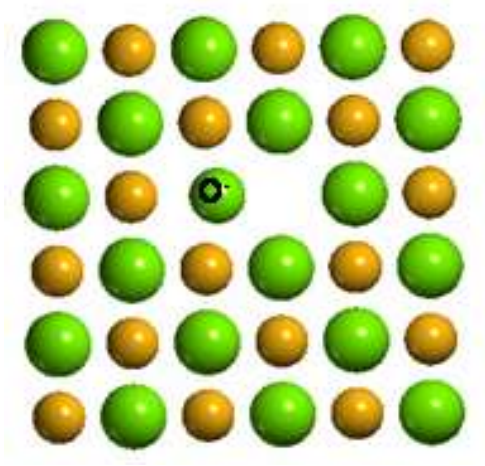

Figure 6. Schematic bidimensional view of a $\mathrm{V}$ centre in $\mathrm{MgO}$. The hole is trapped in correspondence to a cation vacancy.

The $\mathrm{g}$ values measured for $\mathrm{V}^{-}$in $\mathrm{MgO}$ ranges in fact from 2.038 to 2.0003 according to the orientation of the crystal, and the high deviation from $g_{e}$, measured in a particular crystallographic direction is due to the spin orbit coupling (i.e. to the partial admixture of the orbital angular momentum with the spin) which is particularly relevant in the case of oxygen involving centres. Although the structure of the $\mathrm{g}$ tensor is, per se, sufficient to realize that the EPR spectrum is due to an oxygen based centre, more exhaustive is the result obtained introducing ${ }^{17} \mathrm{O}$ into the oxide.

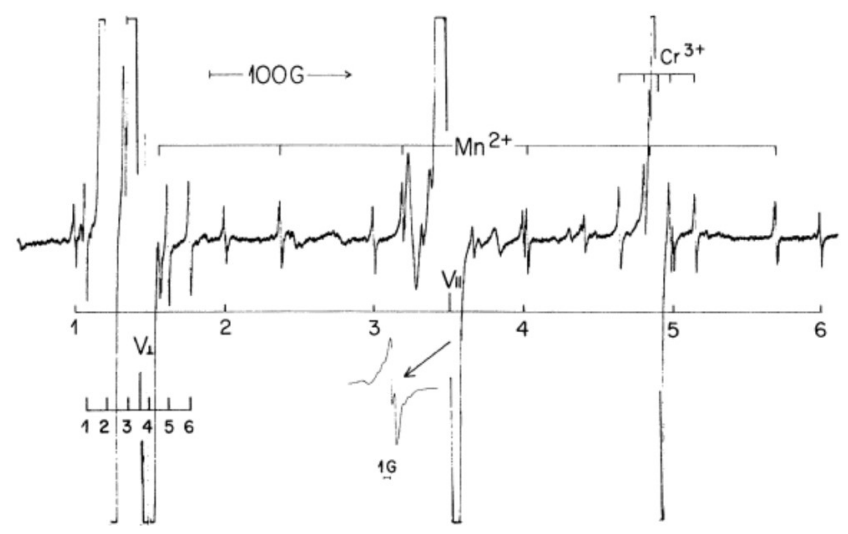

Figure 7. The hyperfine structure of a $\mathrm{V}^{-}$centre in $\mathrm{a}^{17} \mathrm{O}$ enriched $\mathrm{MgO}$ single crystal which contains some paramagnetic impurities $\left(\mathrm{Cr}^{3+}, \mathrm{Mn}^{2+}\right)$. The two labelled sextets (from 1 to 6$)$ are related to the hyperfine interaction of the unpaired electron of the $\mathrm{O}$ - ion (see text) with the ${ }^{17} \mathrm{O}$ nucleus $(I=5 / 2)$. Reproduced from Shoenberg A et al (1971) Phys Rev Lett 27, 1641. 
This isotope has nuclear spin $l=5 / 2$ and generates the hyperfine structure visible in Fig 7 where the two different sextets $(2 \mid+1=6)$ are present, related to two distinct and mutually perpendicular orientations of the center [11]. The measured A values (the separation between the lines of the hyperfine structures) are consistent with the spin density of an unpaired electron confined in a p orbital of oxygen. This result corroborates the chemical description of the trapped hole in terms of an $\mathrm{O}^{-}$ion.

\section{Surface defects as electron traps: a paradigm shift.}

Electrons trapped at point defects on the surface of ionic oxides have been investigated since the late sixties of the past century. This activity eventually led to an evolution of the model introducing a concept of remarkable importance in surface science. It was shown, in fact, that the electron trapping capability concerns a variety of morphological features commonly found at the ionic surface making thus not essential the presence of true surface oxygen vacancies. In earlier studies the above described De Boers model of bulk $F$ center [7], which is based on an electron trapped in a 3-dimensional anion vacancy, was extended to a 2-dimensional surface equivalent. This idea, proposed by A. J. Tench for the surface color (F) centers on irradiated alkali-earth oxides (AEO) [12] regarded the electron as been trapped by the anion vacancy on the (100) surface face of the polycrystalline oxide ( $\mathrm{C}_{4 v}$ type symmetry) with five cations surrounding the vacancy (Figure 8a)

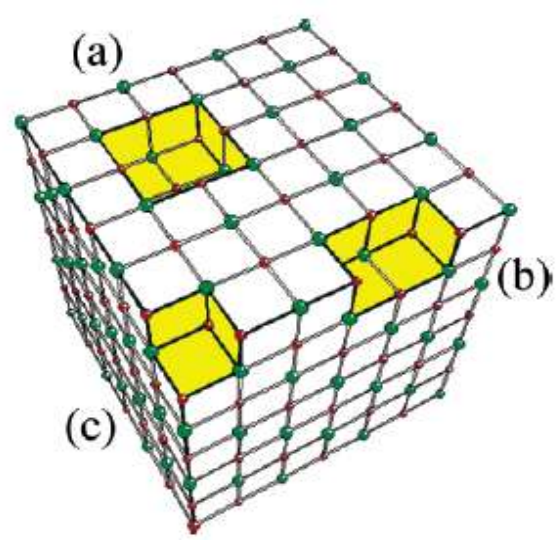

Figure 8. Schematic view of oxygen vacancies at the surface of MgO. a) (100) crystal face (or terrace); b) edge, c) corner. The original Tench model of surface color centers consists in an electron trapped in the terrace vacancy (a).

This model was not based on a direct, self-consistent evidence but was put forward mainly on the basis of the similarity between the EPR spectra of bulk and surface $F$ centers which differ only for the lack of one $\mathrm{Mg}^{2+}$ ion in the latter. However an observation of the same A. J. Tench in 1968, was the starting point which led, about thirty years later, to overcome the 
model described in Figure 8. Tench in fact reported the production of excess electrons centers at the surface of $\mathrm{MgO}$ by contacting the solid with atomic hydrogen [13]. In this way, the solid color turns from white to blue and, in parallel, an EPR spectrum growths up. The latter has strong similarities with the spectra of bulk $\mathrm{F}^{+}$centers, but shows, additionally, a small hyperfine splitting due to the weak interaction of the electron with the parent hydrogen atom $\left({ }^{1} \mathrm{H}, \mathrm{I}=1 / 2\right)$. This result was interpreted on the basis of the oxygen vacancy model, the vacancy being located nearby an hydroxyl $\mathrm{OH}^{-}$group. (Figure 9). Several years later new experiments based on nanostructured, highly crystalline $\mathrm{MgO}$, provided much more resolved EPR spectra and led to a true paradigm shift allowing to conceive new models for the defective sites at the surface of ionic oxides.

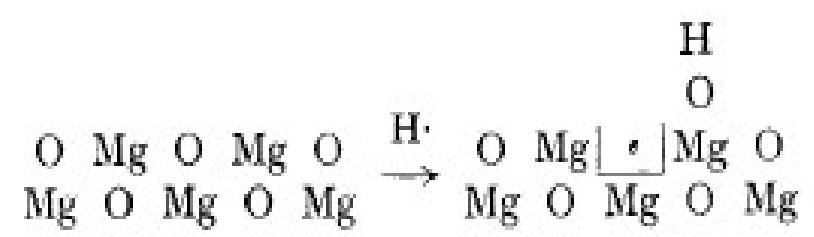

Figure 9. The initial model of $F_{s}(H)^{+}$center. Figure reproduced from D.R. Smith, A. J. Tench (1968) Chem Commun 1113.

Actually, EPR played a leading role in the study surface trapped electron centres. Since analysis of the hyperfine coupling tensor $(A)$ provides a direct measure of spin density on the nuclei nearby the unpaired electron, a realistic chemical and structural description of the surface traps was achieved based on hyperfine interactions [14,15]. However, as this analysis may be insufficient to answer the question on how and where excess electrons are stabilized at surfaces, this information must be complemented with results coming from ab-initio theoretical modelling. The joint use of EPR and theoretical calculations was crucial to unravel the nature of surface electron trapping defects.

The reaction occurring upon contact of atomic hydrogen with the surface of AEOs can be summarized, in the case of $\mathrm{MgO}$, as follows:

$$
\mathrm{Mg}_{n}^{2+} \mathrm{O}^{2-}+\mathrm{H}^{\bullet} \rightarrow \mathrm{Mg}^{2+}{ }_{n}(\mathrm{e}-)(\mathrm{OH}-)
$$

The spontaneous ionisation, at temperatures as low as $77 \mathrm{~K}$, of $\mathrm{H}$ is followed by the formation of excess electrons (trapped in a suitable positive site) and extra protons which become hydroxyl groups reacting with oxygen ions on the surface [15]. The EPR spectrum reported in Figure 10 (upper signal) is characterized by a hyperfine doublet pattern arising from the interaction between the trapped electron and a single proton. An accurate 
investigation of the ${ }^{1} \mathrm{H}$ hyperfine performed by ENDOR (Electron-Nuclear DOuble Resonance) allowed us to detect the hyperfine tensor elements indicating that the local symmetry of the site is lower than axial [16] .The magnitude of these hyperfine couplings clearly indicates a weak electron - proton interaction. In particular, the negligible isotropic component to the hyperfine interaction (i.e., the Fermi contact term) confirms the complete ionisation of the $\mathrm{H}$ atoms upon contact with the ionic surface. Notwithstanding, the presence of the $\mathrm{OH}$ - group is essential not only for a full description of the surface defect but also to confer electron trapping properties to the surface defect. Fortunately, hyperfine interactions are not restricted to the ${ }^{1} \mathrm{H}$ nucleus. The overlap of the excess electron wave function with the charge clouds of surface ions creates further hyperfine interactions with any surface nucleus possessing a magnetic moment and present in relatively high abundance, such as ${ }^{25} \mathrm{Mg}^{2+}(I=5 / 2$ with $10.2 \%$ natural abundance $)$. The analysis of the ${ }^{25} \mathrm{Mg}$ hyperfine pattern proved pivotal in solving the long standing issues surrounding the true nature of the surface excess electron sites.

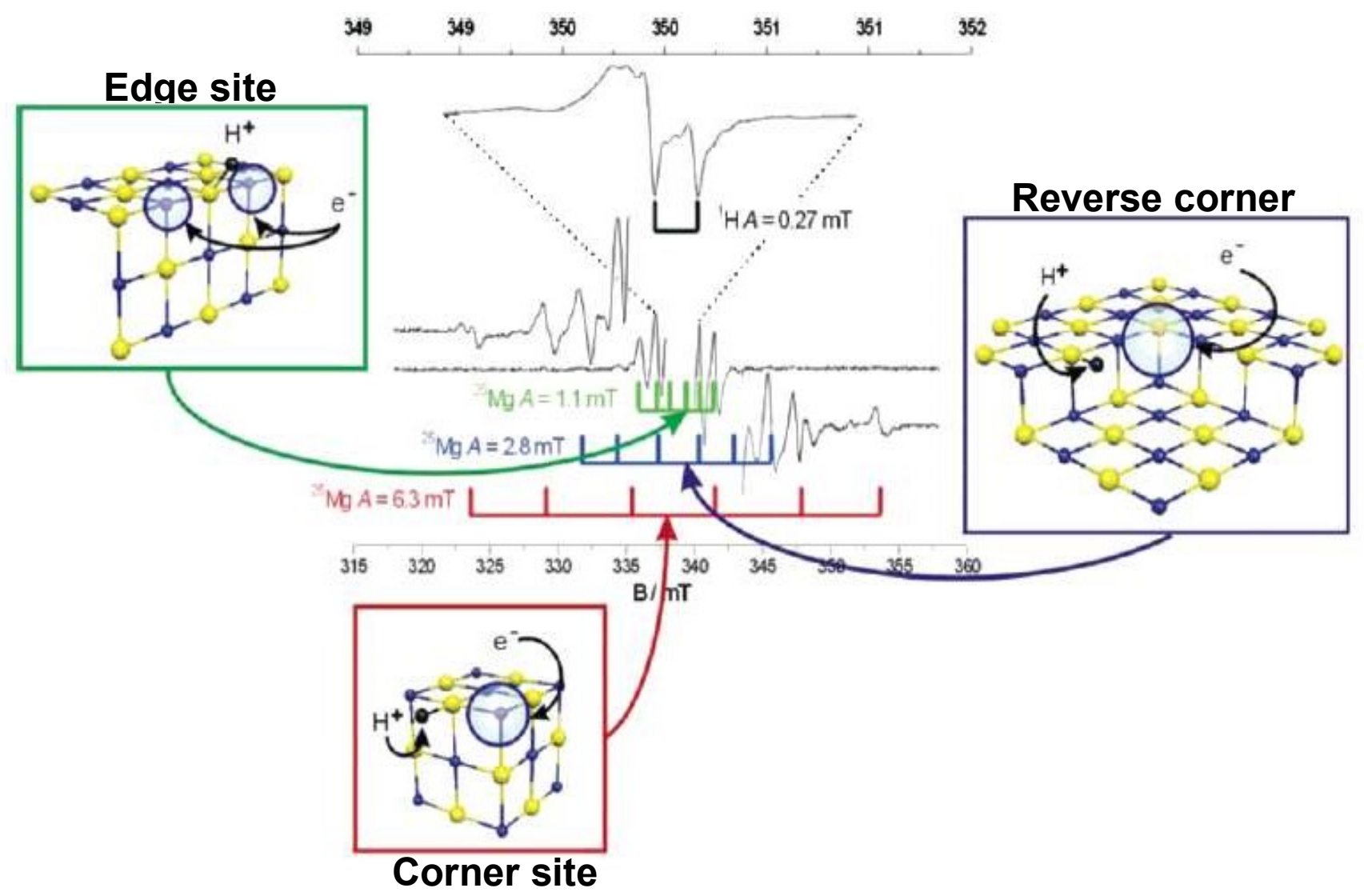

Figure 10. EPR spectrum and atomistic model of three distinct defects sites present on $\mathrm{MgO}$ and capable of electron trapping. The upper signal shows the hydrogen hyperfine doublet. The lower traces are magnifications of the wings of the original signal evidencing the three sextets due to ${ }^{25} \mathrm{Mg}$ hyperfine interaction ( see text, Figure adapted from M. Chiesa et al. (2006) Acc Chem Res 39, 861). 
Three distinct ${ }^{25} \mathrm{Mg}$ hyperfine six-line patterns were experimentally observed, with couplings of $1.1 \mathrm{mT}, 3.0 \mathrm{mT}$ and $6.0 \mathrm{mT}$ (evidenced by the colored stick diagram in Figure 10). Detection of the largest hyperfine sextet $(6 \mathrm{mT})$ prompted us to reconsider the commonly held view of the surface electron traps on $\mathrm{MgO}$ as based on the classical de Boer model $[17,18]$. It is not possible to ascertain a priori the complete number of $\mathrm{Mg}^{2+}$ ions per trapping site, as the statistical probability of finding more than one ${ }^{25} \mathrm{Mg}$ nucleus within a given surface array is small. As a result, the $1.1 \mathrm{mT}$ hyperfine sextet was previously interpreted as arising from the delocalisation of the unpaired electron density over a number of $\mathrm{Mg}^{2+}$ cations constituting a surface oxygen vacancy. This value is larger than that reported for bulk $F^{+}$centers in $\mathrm{MgO}(\cong 0.4 \mathrm{mT})$ [Errore. Il segnalibro non è definito.] but was explained through consideration of the polarizing effect exerted on the unpaired electron by the proton[19]. This effect was reflected in the original nomenclature for these centers, $F_{s}{ }^{+}(H)$, where the subscript $s$ represents surface localization and $(H)$ the presence of a proton in the vicinity of the center [13] . However, the discovery of the new ${ }^{25} \mathrm{Mg}$ hyperfine sextet $(6 \mathrm{mT})$, could not be reconciled with this surface vacancy model. The only reasonable explanation was that the unpaired electron spin density must be localized on a single ${ }^{25} \mathrm{Mg}^{2+}$ cation. Cluster model DFT calculations confirmed this hypothesis and revealed that excess electrons (corresponding to the $6 \mathrm{mT}$ sextet) were indeed stabilized by the large electrostatic potential provided by a single tri-coordinated ion $\left(\mathrm{Mg}_{3 c^{2+}}{ }^{2+}\right.$ at a corner or at a kink with the assistance of a nearby proton [19]. Both the experimentally observed EPR parameters and the energetics of the hydrogen reaction, were accounted for theoretically.

\section{Corner}

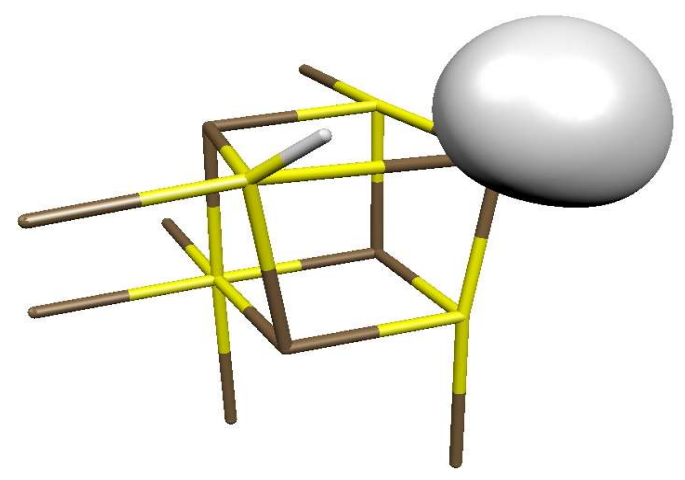


Figure 11. The electron spin density of a trapped electron on the corner of $\mathrm{MgO}$. Figure adapted from Chiesa, et al. (2003) Angew. Chem. Int. Ed. 42, 1759-1761

This result required us to formulate an entirely new model of these centers in terms of $\left(\mathrm{H}^{+}\right)\left(\mathrm{e}^{-}\right)$, electron - proton pairs, bound at morphological surface features such as a corner ion. The two other ${ }^{25} \mathrm{Mg}$ hyperfine patterns $(3.0 \mathrm{mT}$ and $1.1 \mathrm{mT})$ observed experimentally, could also be interpreted and explained using the new models. Again theoretical calculations showed that the $3 \mathrm{mT}$ hyperfine pattern could be reconciled with a $\left(\mathrm{H}^{+}\right)\left(\mathrm{e}^{-}\right)$ pair localised at the intersection of two steps. It transpires that this morphological feature, also known as a reverse corner, is an important defect on $\mathrm{MgO}$ responsible for a number of interesting reactions, from the heterolytic dissociation of $\mathrm{H}_{2}$ to the stabilization of alkali metal atoms [20]. Far more problematic was the assignment of the $1.1 \mathrm{mT}{ }^{25} \mathrm{Mg}$ hyperfine pattern, which is also the most intense pattern. The magnitude of this coupling could be equally reproduced, by ab-initio calculations, using two drastically different models; the classical surface anion vacancy model, $F_{s}{ }^{+}(\mathrm{H})$ center, and the $\left(\mathrm{H}^{+}\right)\left(\mathrm{e}^{-}\right)$pairs model localized at surface edges and steps [21]. While the energetic and electrostatic considerations clearly favour the second model, the final definitive assignment was only achieved by performing a number of experiments using $\mathrm{MgO}$ surface enriched with ${ }^{17} \mathrm{O}(\mathrm{I}=$ $5 / 2$ ). Enrichment was achieved by repeated hydration/dehydration cycles of high surface area $\mathrm{MgO}$ using $\mathrm{H}_{2}{ }^{17} \mathrm{O}$. In this way the unpaired electron spin density distribution could be monitored over the constituent $\mathrm{O}^{2-}$ ions of the surface electron trap [22]. The EPR spectrum of ${ }^{17} \mathrm{O}$ enriched $\mathrm{MgO}$ containing excess electrons is shown in Figure 12.

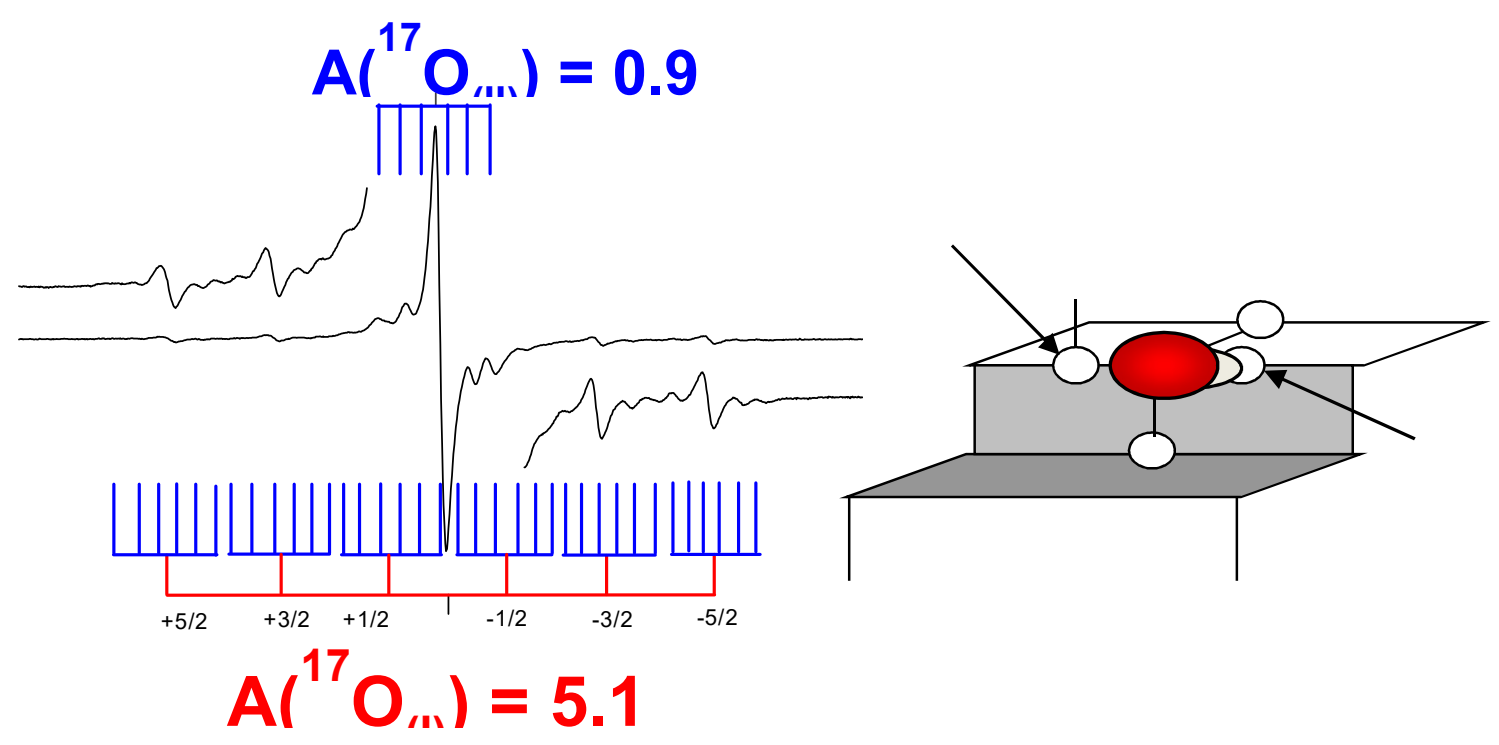


Figure 12. EPR spectrum of the edge defect site recorded using ${ }^{17} \mathrm{O}$ enriched $\mathrm{MgO}$ (left) and schematic view of the site (right). In the scheme the red ellipse represents the trapped electron. In the EPR spectrum the two distinct hyperfine structures related to the interaction with $\mathrm{O}_{(\mathrm{I})}$ (the hydroxilic oxygen) and $\mathrm{O}_{(\mathrm{II})}$ are evidenced.

The spectrum is dominated by two ${ }^{17} \mathrm{O}$ hyperfine sextets arising from the interaction of the unpaired electron with two, magnetically inequivalent, ${ }^{17} \mathrm{O}$ nuclei which possess different spin densities due to the preferential polarization of the trapped electron towards one of the two nuclei. This polarisation is created by the nearby surface $\mathrm{OH}^{-}$group, which has the larger ${ }^{17} \mathrm{O}$ hyperfine coupling $\left(\mathrm{A}^{17} \mathrm{O}_{(\mathrm{I})}\right.$ in Figure12) while the smaller coupling $\left(\mathrm{A}^{17} \mathrm{O}_{(\mathrm{II})}\right)$ belongs to the surface $\mathrm{O}^{2-}$ lattice anions. This intuitive assignment was confirmed by ab initio calculations of the ${ }^{17} \mathrm{O}$ hyperfine tensors, which revealed that only the $\left(\mathrm{H}^{+}\right)\left(\mathrm{e}^{-}\right)$pairs model, based at surface steps or edges is consistent with the experimental data, since the ${ }^{17} \mathrm{O}$ hyperfine couplings for the $\mathrm{F}_{S}{ }^{+}(\mathrm{H})$ model were far too small.

To summarize, at least three different sites have been identified on the MgO surface which are able to spontaneously ionise $\mathrm{H}$ atoms and to stabilize the resulting products in the form of $\left(\mathrm{H}^{+}\right)\left(\mathrm{e}^{-}\right)$pairs, according to eq. (3); these sites (e.g. edge, corner and reverse corner sites) are illustrated in Figure 10. The ab initio calculations show that the $\left(\mathrm{H}^{+}\right)\left(\mathrm{e}^{-}\right)$centre on $\mathrm{MgO}$ (reverse corner) is a deep trap for the electron which is bound by $3.71 \mathrm{eV}$ and gives rise to two intense electronic transitions in the visible at $2.07 \mathrm{eV}$ and $2.39 \mathrm{eV}$ [Errore. II segnalibro non è definito.]. The same is true for corner sites [19]. The described findings provides a new concept in the area of surface point defects in oxides introducing the idea that discrete morphological features, naturally present on surfaces, have properties (Madelung field, charge trapping ability) parallel to those of typical bulk point defects. In this perspective a discrete array of ions in particular locations of a nanostructured surface can be seen as a true and original surface point defect.

The new picture on the nature of the excess electron trapping sites on the MgO surface does not imply that oxygen anion vacancies are irrelevant and absent in the surface chemistry of $\mathrm{MgO}$. Oxygen vacancies are thought to be key factors not only in the nucleation, growth and stabilization of supported metal clusters but also in their chemical activation in catalytic reactions [23]. Since low coordinated sites are quite abundant on polycrystalline powders, the original vacancy model plays a negligible role in electron trapping at the surface of these materials. The concentration of oxygen vacancies at a (100) face of $\mathrm{MgO}$ at the equilibrium is, in fact, intrinsically low as it depends exponentially on the formation enthalpy of the defect (the higher the entalphy, the lower the defect concentration). The lattice energy of an ionic solid such as $\mathrm{MgO}$, based on an alternance 
of divalent ions, is rather high. Consequently, the formation enthalpy of an isolated oxygen vacancy at the 100 face is also high and can be estimated around $6.35 \mathrm{eV}(96 \%$ of the corresponding value for a bulk vacancy). In other words the expected number of surface oxygen vacancies at the $\mathrm{MgO}$ terrace, at room temperature, is comparable to that, extremely small, of bulk vacancies.

The substantial absence of oxygen vacancies on the MgO terraces at thermodynamic equilibrium does not mean that it is impossible to generate such kind of point defect using suitable methods. A significant improvement in the knowledge of oxygen vacancies came, in fact, from the use of ultrathin oxide films epitaxially grown on metal substrates. In order to observe oxygen vacancies on such systems, however, it was necessary to artificially create these defects by electron bombardment. The nature of these defect centres on bombarded $\mathrm{MgO}$ films could thus be investigated by a number of techniques including STM and EPR [24]. In particular EPR measurements allowed not only the attainment of the magnetic quantities but also, in conjunction with STM studies, that of the geometrical arrangement corresponding to the observed signal [25]. The joint use of the two techniques has produced in fact strong evidence for the nature of surface oxygen vacancies showing their preferential formation on steps and edges (Figure 8b) rather than on terraces [26]. The measurements have also shown that the number of $F_{\mathrm{s}}$ centers in the pristine, as grown stoichiometric films is very small, below the detection limit of an EPR spectrometer as expected on the basis of their high formation energy.
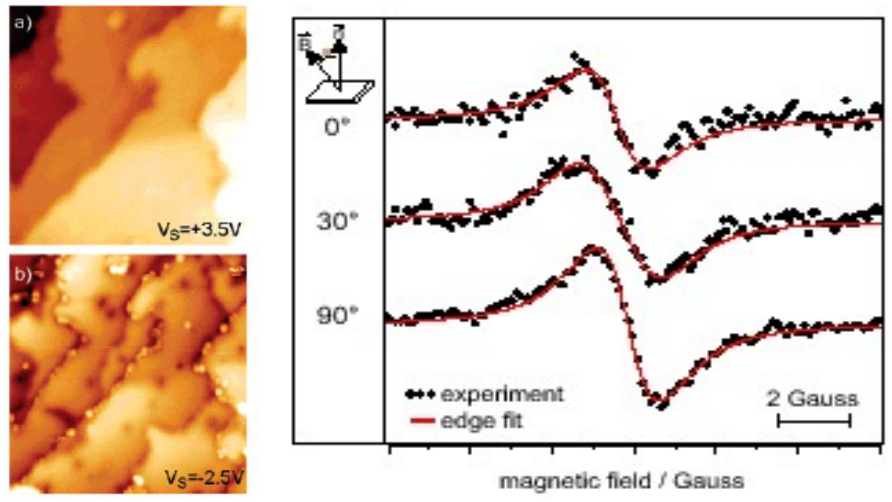

Figure 13. Left: STM images at different sample bias of a MgO (001) film grown on $\mathrm{Ag}(100)$ and submitted to electron bombardment. Right: EPR spectra and related simulation of a $\mathrm{MgO}(001)$ film grown on $\mathrm{Mo}(001)$. Adapted from Sterrer, et al. (2005) Phys.Rev Lett 94, 186101 and Sterrer et al. (2006) J Phys Chem B 110, 46-49. 
The EPR spectra of $\mathrm{F}$ centers obtained are shown in Fig. 13B. The signal is quite isotropic and shows, as expected, slight shifts of the resonant field (hence of the $g$ value, Equation 1) varying the orientation of the single crystalline film.

\section{Surface trapping sites for charge carriers.}

Charge carriers formation, stabilisation and reactivity at the surface have great importance in the photochemistry of oxides. Light induced charge separation, in fact, is relevant for a series of applications aiming to exploit the photon energy of electromagnetic radiation. The surface of metal oxides in particular are able to stabilize, either independently or simultaneously, electrons and holes. Both the conversion of photon energy into electric energy (photovoltaic) and the photochemical applications such as photocatalysis or water photosplitting have a common starting point, which consists in the charge separation induced by a photon with above band gap energy. Photon absorption in fact generates a pair of carriers constituted by an electron and an hole. The two carriers after formation can either recombine (dissipation of photon energy) or move into the crystal. For the mentioned photochemical applications it is of vital importance the migration of the carriers at the surface of the solid where they find a suitable site for their stabilisation (in other words a surface defect) and can thus entail chemical interactions with the surroundings. Monitoring the presence, the stabilisation and the reactivity of charge carriers at oxides surfaces is therefore a crucial problem for photochemical and photophysical applications of oxides $[27,28]$.

\subsection{Insulating ionic oxides}

Irradiating fully dehydrated $\mathrm{MgO}$ with photons having enough energy to cover the band gap (7.8 eV), surface stabilised charge carriers are observed by EPR as shown in Fig. 14. The feature at around $g_{e}$ is due to a trapped electron while the feature at lower field is the perpendicular component of the signal of a trapped hole. Both centres (as demonstrated by their fast reactivity with molecules admitted in the gas phase) are indeed localized at suitable sites at the surface of the oxide nanocrystals. The two paramagnetic centres are metastable and the corresponding signals decay, due to recombination, in the time scale of some hours at 77K, as shown in Fig.14b. Since the detailed features of the signal at high field are similar to those of the signal observed upon contacting atomic hydrogen with $\mathrm{MgO}$ and have been discussed before (Section 5), in the present Section the description of EPR of surface hole centres only will be tackled. 
If stabilisation of an hole occurs in some particular location of the crystal, the hole centre is usually observable by EPR and a rich family of such defects, $V$ centres, has been identified. As discussed in Section 4 localized hole on an anionic site of an oxide like $\mathrm{MgO}$ can therefore be described in chemical terms as a (paramagnetic) $\mathrm{O}^{-}$ion and its formation under irradiation, leading to the EPR spectrum in Fig.14, can be written as:

$$
\mathrm{Mg}^{2+}+\mathrm{O}^{2-}+\mathrm{h} v \rightarrow \mathrm{Mg}^{2+}\left(\mathrm{e}^{-}\right)+\mathrm{O}^{2-}(\mathrm{h}+) \rightarrow \mathrm{Mg}^{2+}(\mathrm{e}-)+\mathrm{O}^{-}
$$

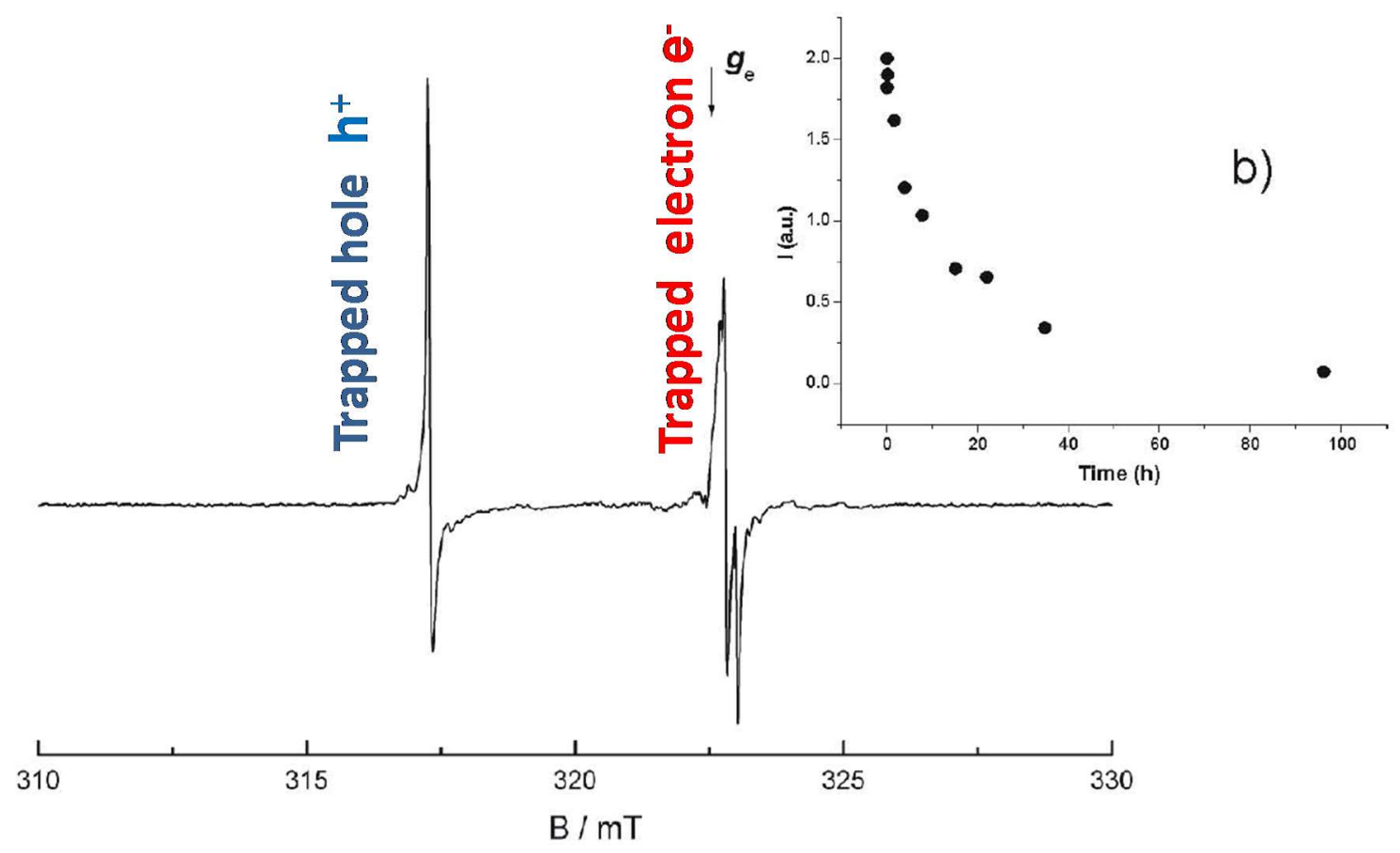

Figure 14. a) CW-EPR of trapped electron (r.h.s.) and hole centres (I.h.s.) generated at the surface of polycrystalline $\mathrm{MgO}$ by $\mathrm{X}$ ray irradiation Spectrum recorded at $77 \mathrm{~K}$. b) Intensity decrease with time of the two EPR signals in Fig.1a. Figure reproduced from Chiesa et al.(2010) Appl Mag Res 37, 615-618

The EPR feature of the surface trapped hole on polycrystalline MgO (Fig.14, low field) is actually the perpendicular component of an axial signal whose parallel feature is hidden by the trapped electron signal around ge. Recording the same spectrum at higher microwave power induces saturation of the trapped electron EPR line so that the whole $\mathrm{O}^{-}$ signal can be appreciated. This signal shows the profile of an axial signal (Fig. 15) with $g_{\perp}$ 
$>\mathrm{g} \| \cong \mathrm{ge}_{\mathrm{e}}\left(\mathrm{g}_{\perp}=2.0358 ; \mathrm{g} \|=2.0025\right)$. Such a signal is typical of a hole trapped onto an oxygen ion in a site of relatively low symmetry like a surface site. The presence of a hole in an oxide system does not necessarily produce, in spite of the paramagnetic nature of $\mathrm{O}^{-}$, an EPR spectrum. There are at least two cases corresponding to EPR silent $\mathrm{O}^{-}$ions. The first occurs in the case of highly mobile holes in fast random motion and the second in the case of a highly symmetric environment around the hole itself. The expected structure of the $g$ tensor for the $\mathrm{O}^{-}$radical (electron configuration $2 \mathrm{px}^{2}, 2 \mathrm{py}^{2}, 2 \mathrm{p}^{2}$ ) ion in the case of axial symmetry and neglecting second order terms is:

$$
\begin{aligned}
& g_{z z}=g \| \approx g_{e} \\
& g_{x x}=g_{y y}=g_{\perp}=g_{e}+2 \lambda / \Delta E
\end{aligned}
$$

where $\lambda$ is the spin orbit coupling constant and $\Delta \mathrm{E}$ is the difference between the ground and first excited electronic state roughly corresponding to the separation between the $2 p_{z}$ and the other two $p$ orbitals induced by crystal field effects (see Scheme 1).

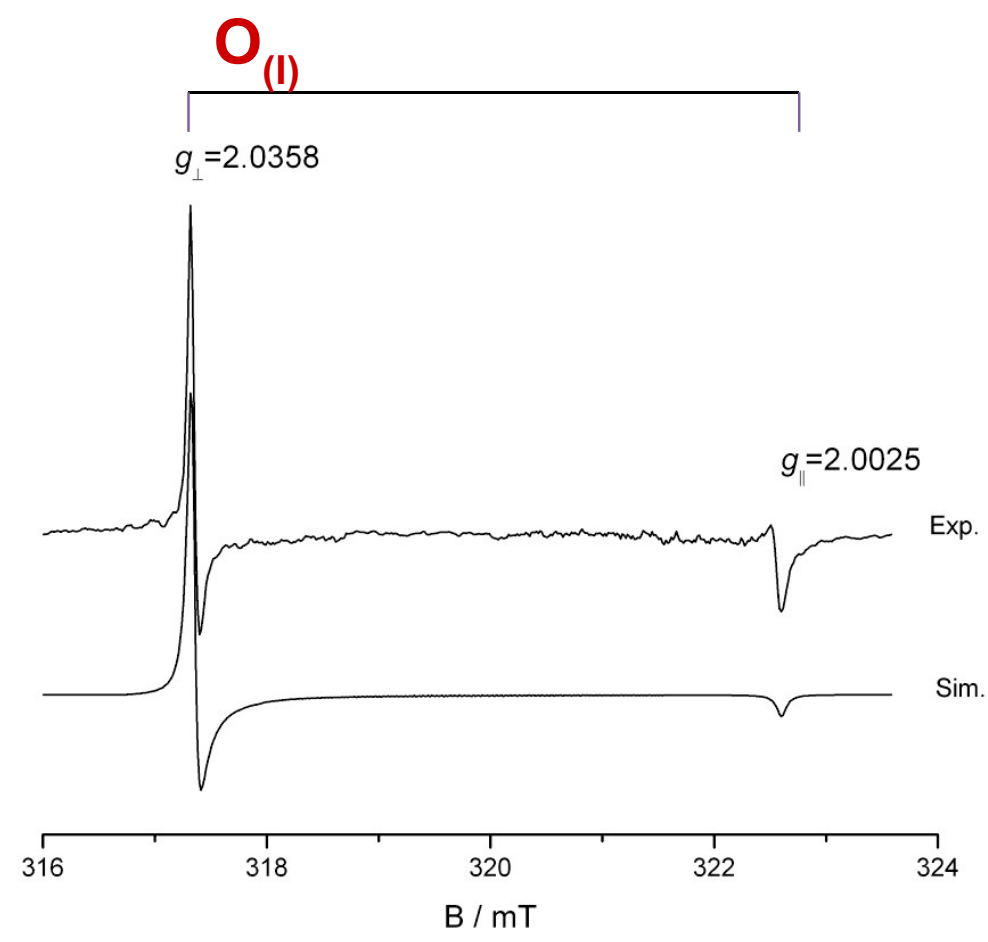

Figure 15. EPR spectrum and related simulation of the trapped hole at the surface of polycrystalline MgO. The trapped electron signal has been saturated using an high microwave power. Traces of that signal are still appreciable in the experimental spectrum. 
$\Delta \mathrm{E}$ directly depends on the magnitude and symmetry of the electrostatic field felt by the anion.

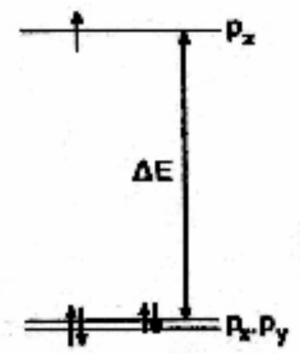

\section{Scheme 1.}

Information from the spectrum in Fig.15 are limited to the $\mathrm{g}$ tensor as ${ }^{16} \mathrm{O}$ (the dominant isotope of oxygen with an abundance of $99.9 \%$ ) has nuclear spin I=0 and does not generate any hyperfine structure. However recent experimental activity led us to obtain isotopic enrichment of the $\mathrm{MgO}$ surface with ${ }^{17} \mathrm{O}$ by successive hydration and dehydration cycles of the sample using $\mathrm{H}_{2}{ }^{17} \mathrm{O}$ at an extent sufficient to observe the hyperfine structure of the ${ }^{17} \mathrm{O}^{-}$ion [29] as shown in Fig 16.

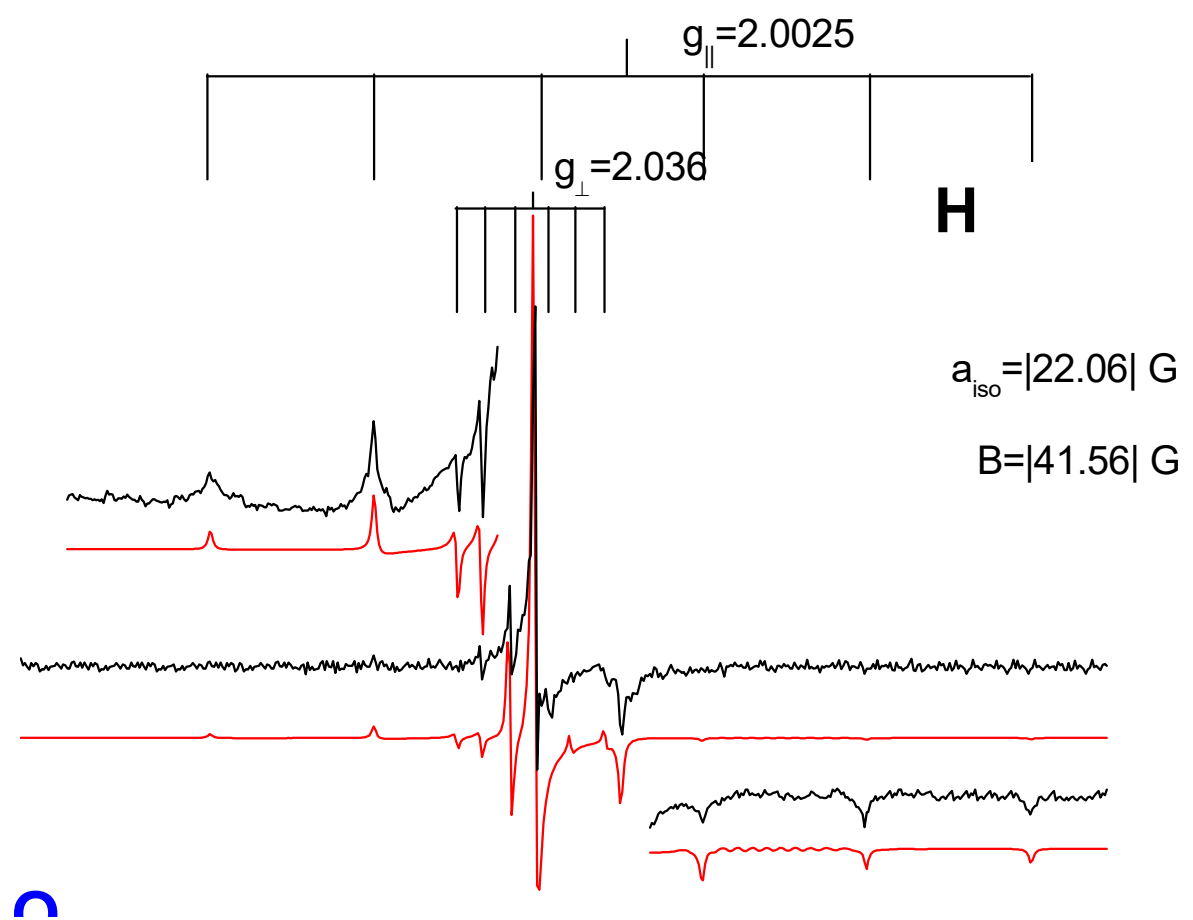


Figure 16. Experimental and computer simulated EPR spectra of an X-ray irradiated polycrystalline MgO sample previously enriched with ${ }^{17} \mathrm{O}$.

Since the isotopic enrichment is limited (about $10 \%$ ), the spectrum in Fig. 5 is the superposition of the signal of two isotopomers $\left({ }^{16} \mathrm{O}^{-}\right.$and $\left.{ }^{17} \mathrm{O}^{-}\right)$. For each principal direction of the $\mathbf{g}$ tensor, the signal of ${ }^{17} \mathrm{O}^{-}(I=5 / 2)$ is split into $2 I+1=6$ lines having separation $\mathrm{A}_{\perp}=1.9 \mathrm{mT}$ and $\mathrm{A} \|=10.5 \mathrm{mT}$ respectively. The hyperfine matrix can thus be analyzed deriving the isotropic aiso $(-2.2 \mathrm{mT})$ and anisotropic dipolar $(-8.2 \mathrm{mT})$ terms. The spin density $\rho$ on the oxygen orbitals, obtained comparing the above values with the tabulated atomic values for the ${ }^{17} \mathrm{O}$ isotopes gives $\rho^{2 s}=0.012$ and $\rho^{2 p}=0.820$ respectively. The total spin density on the $\mathrm{O}^{-}$ion is therefore $\rho^{\text {tot }}=0.832$ [29]. The spin density values indicate that the unpaired electron wave function has a small $s$ character, probably due to some degree of spin polarization, and that the unpaired electron spin density is mainly localized in a $p$ orbital of oxygen as expected for an $\mathrm{O}^{-}$radical ion and as actually found for bulk examples of $V_{0}$ centers [28]. The axial symmetry of both $g$ and $A$ tensors and the comparison of their experimental values with those calculated by DFT unambiguously indicate that the hole is stabilized on the surface site having the lowest coordination. This is a three-coordinated oxygen ion at the corner of the cubic nanocrystal $\left(\mathrm{O}^{-}\right.$зc $)$as shown in Scheme 2.

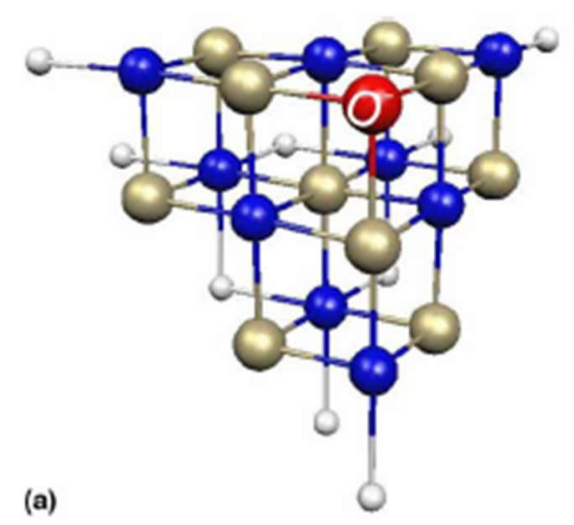

Scheme 2. The hole trapping site $\left(\mathrm{O}^{-}\right)$at the surface of $\mathrm{MgO}$.

As mentioned above theoretical modeling of $\mathrm{O}^{-}$ions at low-coordinated sites substantially confirms these conclusions. The $\mathrm{C} 3 \mathrm{v}$ symmetry of the $\mathrm{O}^{-}$ion at the corner site leads to the correct axial symmetry of the $g$ and hyperfine tensors, as experimentally observed, while the lower local symmetry of the $\mathrm{O}^{-}$ion at the edge site leads to an orthorhombic g-tensor. The agreement between experimental and computed g-tensor 
elements is qualitatively correct since in all cases it is possible to observe a relatively small positive shift from the free electron $g$ value $\left(g_{e}=2.0023\right)$ for the $z$ component while a larger deviation in the same direction is found for the $\mathrm{x}$ and $\mathrm{y}$ components. Also hyperfine coupling constants are accurately computed. The experimental isotropic component is somewhat underestimated by calculations but the computed dipolar matrix elements almost coincide with the experimental values. Mulliken population analysis attributes a total spin density of 0.9 to the $\mathrm{O}^{-}$ion. Considering the tendency of DFT calculations to produce delocalized spin states, this is a good evidence for the localized nature of the trapped hole.

\subsection{Semiconducting oxides}

For the reasons explained at the beginning of this section, the phenomena of charge separation are more important in the case of semiconducting oxides than in that of insulators. Semiconducting oxides usually contain transition ions capable of displaying multiple oxidation states. This implies that some semiconducting oxides are also reducible oxides. Many of the most investigated oxides for catalytic applications (eg $\left.\mathrm{CeO}_{2}\right)$ or photocatalysis (eg, $\mathrm{TiO}_{2}$ ) are reducible oxides and tend to lose oxygen when annealed under vacuum. This phenomenon generates oxygen vacancies and, at the same time, reduced states (i.e. excess electrons ). Unlike the case of insulating oxides, however, the excess electrons in these oxides tend to stabilize in the orbitals of the reducible metal ion. Though often invoked, the presence of electrons trapped in the volume of the oxygen vacancies (i.e. the presence of $F$ centers) in reducible oxides, have never found a strong and indisputable evidence. The study of surface oxygen vacancies in systems such as $\mathrm{TiO}_{2}$ and $\mathrm{CeO}_{2}$ has been tackled by surface science in recent years. As to the EPR technique a number of paramagnetic defects, based on oxygen vacancies but localized in the bulk of $\mathrm{TiO}_{2}$ have been recently reported by the group of Halliburton. Among these defects the more important are $\mathrm{Ti}^{3+} \mathrm{V}_{\mathrm{O}}$ (an excess electron associated to an oxygen vacancy) and $\mathrm{Ti}^{3+} \mathrm{V}_{\mathrm{O}} \mathrm{Ti}^{3+}$ (two electrons associated with an oxygen vacancy that form a spin triplet with $S=1$ ). In all these cases the EPR evidence clearly indicates that the excess electrons are associated with the $d$ orbitals of titanium ions .

In the case of polycrystalline materials the formation of charge carriers under irradiation has also been followed by EPR. Before irradiation the EPR spectrum of pristine titania has a flat base line. Irradiation of nanocrystalline anatase with UV light produces the EPR spectrum shown in Fig. 17 which shows evidence of charge separation and trapping. 
Electrons, in these conditions, are trapped on titanium ions. The signal at high field (right hand side of the spectrum in Fig. 17) is in fact similar to those shown by $\mathrm{Ti}^{3+}$ containing molecular compounds or by titanium dioxide containing excess electrons. The signal (characterized by $\mathrm{g}_{\|}=1.962$ and $\mathrm{g}_{\perp}=1.992$ ) is thus unambiguously due to $\mathrm{T}^{3+}$ ions.

The signal at low field is consequently due to trapped holes. The signal is made up by the contribution of at least two similar species with slightly different parameters. At the microwave power employed to record the spectrum in Fig. 17 one of these species is dominant and has $\mathrm{g}_{1}=2.027, \mathrm{~g}_{2}=2.015, \mathrm{~g}_{3}=2.003$. The signal has a rhombic symmetry (three distinct $g$ values) reflecting the symmetry of the paramagnetic site. These values are similar however to those previously illustrated for the hole on $\mathrm{MgO}$ surface' in line with the fact that, for both oxides, the hole itself is stabilized by an oxygen anion producing an $\mathrm{O}^{-}$. The hole are trapped at the surface since they easily react with hole scavengers like molecular hydrogen.

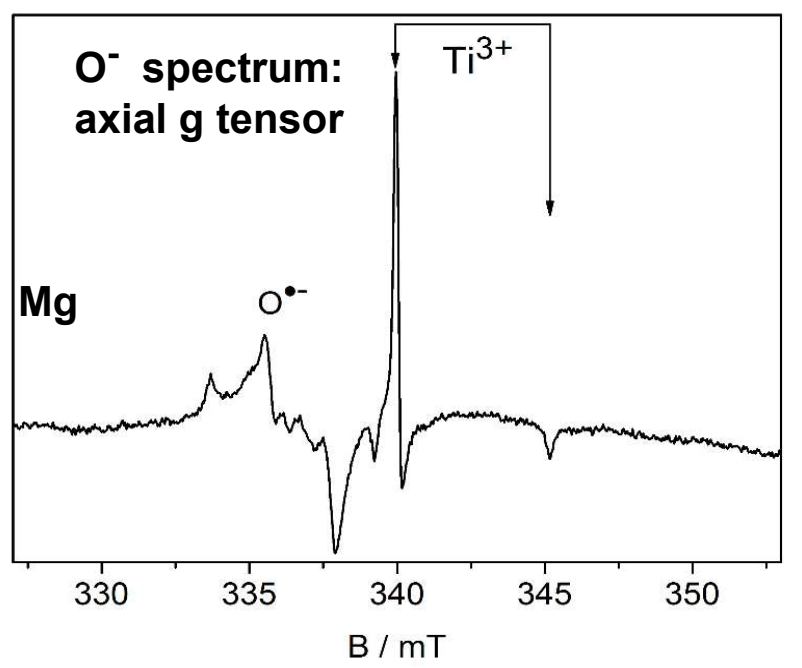

Figure 17. EPR spectrum recorded at $77 \mathrm{~K}$ of titanium dioxide (anatase) under irradiation with UV light. The signals of the trapped electron and hole are clearly discernible.

\section{Conclusions}

In the present chapter we have discussed some applications of the Electron Paramagnetic Resonance (EPR) technique to the study of point defects at the surface of oxides. This technique plays an important role in the study of paramagnetic defects (i.e. centers containing umpired electrons)in that it has an unsurpassed ability in monitoring the localization (or delocalization) of the electron spin density. The attention has been initially 
paid to the morphological defects at the surface of alkaline earth oxides (whose features have been thoroughly described via the coupling of EPR with advanced computational chemistry ) and then to the defects due to the surface trapping of charge carriers in particular when these are generated by photo excitation. Once again the EPR technique is extremely accurate in monitoring both trapped electrons and trapped holes at the surface of oxides. While the holes, since they tend to localize on surface oxygen sites, have similar features for all types of oxide, the electrons tend to be trapped at surface positive morphological defects in the case insulating ionic oxide while they result stabilized in dorbitals in the case of transition metal oxides.

\section{References}

[1] Brustolon M, Giamello E, Eds (2009) Electron Paramagnetic Resonance. A practitioner toolkit. John Wiley \& Sons Inc.

[2] Spaeth J-M, Overhof H. (2003) Point Defects in Semiconductors and Insulators, Springer, Berlin,

[3] Schmidt J, Risse T, Hamann H, Freund H J (2002) J. Phys. Chem. B 116, 10861.

[4] Futako, W.; Umeda, T.; Nishizawa, M.; Yasuda, T.; Isoya, J.; Yamasaki S (2002), J. Non-Crystalline.

Solids, 299, 575.

[5] Sterrer, M.; Fischbach; E., Risse, T.; Freund, H. J. Phys. Rev. Lett. 2005, 94 , 186101.

[6] Chiesa M, Che M, Giamello E (2010) EPR Characterization and Reactivity of Surface Localized Inorganic Radicals and Radical lons. Chem Rev

[7] De Boer, J. H. Rec. Trav. Chim., 1937, 301.

[8] Seidel H, Wolf H C (1968) EPR and ENDOR Spectroscopy of Color Centers in Alkali Halide Crystals in Physics of Color Centres, Beall Fowler W Ed. Academic Press, New York.

[9] Henderson B, Wertz J E (1968) Defects in the alkaline earth oxides. Adv Phys 17, 749.

[10] Wertz J.E, Auzins, P, Griffiths JHE, Orton JW (1959) Spin resonance studies of defects in Magnesium Oxide, Discuss Faraday. Soc 28, 136.

[11] Shoenberg A, Suss JT, Szapiro S, Luz Z (1971) Oxygen-17 hyperfine interaction of $V_{F}$ centres in MgO Phys Rev Lett 27, 1641.

[12] Tench A J, Nelson R L (1968) Paramagnetic defects associated with hydrogen absorbed on the surface of magnesium and calcium oxide J. Colloid Interface Sci. 26, 364-373

[13] D.R. Smith, A. J. Tench Chem. Commun. 1968, 1113.

[14] Giamello E, Paganini M C, Murphy D M, Ferrari A M, Pacchioni G (1997) A combined EPR and quantum chemical approach to the structure of surface $\mathrm{Fs}(\mathrm{H})$ centers on MgO. J. Phys. Chem. 101, 971-982

[15] Chiesa M, Paganini M C, Giamello E, Di Valentin C, Pacchioni, G (2006) Electron traps on oxide surfaces: $\left(\mathrm{H}^{+}\right)\left(\mathrm{e}^{-}\right)$pairs stabilized on the surface of ${ }^{17} \mathrm{O}$ enriched $\mathrm{CaO}$, ChemPhysChem, 7, 728-734.

[16] Murphy D M, Farley R D, Purnell I J, Rowlands C, Yacob A R, Paganini M C, Giamello E (1999) Surface defect sites formed on partially and fully dehydrated MgO; An EPR/ENDOR study J. Phys. Chem. B 103, 1944-1953

[17]Chiesa M, Paganini M C, Giamello E, Murphy D M, Di Valentin C, Pacchioni G (2006) Acc. Chem. Res. 39, 861

[18] Chiesa M, Paganini M C, Giamello E, Di Valentin C, Pacchioni G.(2003) First evidence of a single-ion electron trap at the surface of an ionic oxide. Angew Chem Int Ed 42, 1759-1761.

[19] Ricci D, Di Valentin C, Pacchioni G, Sushko PV, Shluger A L, Giamello E (2003) Paramagnetic defect centers at the MgO surface. An alternative model to oxygen vacancies, J Am Chem Soc 125, 738.

[20] Chiesa M, Giamello E, Di Valentin C, Pacchioni G, Sojka Z, Van Doorslaer S (2005) The nature of the chemical bond between metal atoms and oxide surfaces: new evidences from spin density studies of $K$ atoms on alkaline earth oxides, J Am Chem Soc 127, 16935-16944.

[21] Chiesa M, Paganini M C, Spoto G, Giamello E, Di Valentin C, Del Vitto A, Pacchioni G, (2005) Single electrons trapped at the surface of polycrystalline MgO: assignment of main trapping sites J Phys Chem B, $109,7314-7322$ 
[22] Chiesa M, Martino P, Giamello E, Di Valentin C, Del Vitto A, Pacchioni G (2004) Local environment of electrons trapped at the $\mathrm{MgO}$ surface: spin density on the oxygen ions from ${ }^{17} \mathrm{O}$ hyperfine coupling constants $\mathrm{J}$ Phys Chem B 108, 11529-11534.

[23] Abbet S, Riedo E, Brune H, Heiz U, Ferrari A M, Giordano L, Pacchioni G (2001) Identification of defect sites on $\mathrm{MgO}(100)$ thin films by decoration with $\mathrm{Pd}$ atoms and studying $\mathrm{CO}$ adsorption properties, $\mathrm{J}$ Am Chem Soc 123, 6172-6178.

[24] Sterrer M, Heyde M, Novicki M, Nilius N, Risse T, Rust H-P, Pacchioni G, Freund H-J (2006) Identification of color centers on MgO(001) thin films with Scanning Tunneling Microscopy, J Phys Chem B, $110,46-49$

[25] Sterrer M, Fischbach E, Risse T, Freund H-J (2005) Geometric characterization of a singly charged oxygen vacancy on a single crystalline (001) MgO film by EPR spectroscopy, Phys Rev Lett 94, 186101 [26] Sterrer M, Heyde M, Novicki M, Nilius N, Risse T, Rust H-P, Pacchioni G, Freund H-J (2006) Identification of color centers on $\mathrm{MgO}(001)$ thin films with Scanning Tunneling Microscopy, J Phys Chem B, 2006, 110, 46-49.

[27] Howe R F, Grätzel M (1987) EPR study of hydrated anatase under UV irradiation J Phys Chem 91, 3906

[28] Chiesa M, Paganini M C, Giamello E (2010) EPR of charge carriers stabilized at the surface of metal oxides Appl Mag Res 37, 615-618

[29] Chiesa M , Martino P, Giamello E, Di Valentin C, Del Vitto A, Pacchioni G (2004Chem Phys Lett. 